\title{
A large National Institute for Health Research (NIHR) Biomedical Research Centre facilitates impactful cross-disciplinary and collaborative translational research publications and research collaboration networks: a bibliometric evaluation study
}

\author{
Vasiliki Kiparoglou ${ }^{1,2}$, Laurence A. Brown $n^{3,4}$, Helen McShane ${ }^{1,5}$, Keith M. Channon ${ }^{1,6}$ and \\ Syed Ghulam Sarwar Shah ${ }^{1,7^{*}}$ (D)
}

\begin{abstract}
Background: The evaluation of translational health research is important for various reasons such as the research impact assessment, research funding allocation, accountability, and strategic research policy formulation. The purpose of this study was to evaluate the research productivity, strength and diversity of research collaboration networks and impact of research supported by a large biomedical research centre in the United Kingdom (UK).

Methods: Bibliometric analysis of research publications by translational researchers affiliated with the National Institute for Health Research (NIHR) Oxford Biomedical Research Centre (BRC) from April 2012 to March 2017.

Results: Analysis included 2377 translational research publications that were published during the second 5-year funding period of the NIHR Oxford BRC. Author details were available for $99.75 \%$ of the publications with DOls (2359 of 2365 with DOls), and the number of authors per publication was median 9 (mean $=18.03, S D=3.63$, maximum $=2467$ authors). Author lists also contained many consortia, groups, committees, and teams ( $n=165$ in total), with 1238 additional contributors, where membership was reported. The BRC co-authorship i.e., research collaboration network for these publications involved 20,229 nodes (authors, of which 1606 nodes had Oxford affiliations), and approximately 4.3 million edges (authorship linkages). Articles with a valid DOls (2365 of 2377, 99.5\%) were collectively cited more than 155,000 times and the average Field Citation Ratio was median 6.75 (geometric mean $=7.12$ ) while the average Relative Citation Ratio was median 1.50 (geometric mean $=1.83$ ) for the analysed publications.
\end{abstract}

Conclusions: The NIHR Oxford BRC generated substantial translational research publications and facilitated a huge collaborative network of translational researchers working in complex structures and consortia, which shows success across the whole of this BRC funding period. Further research involving continued uptake of unique persistent

*Correspondence: Sarwar.Shah@ouh.nhs.uk

${ }^{1}$ NIHR Oxford Biomedical Research Centre, John Radcliffe Hospital, Oxford University Hospitals NHS Foundation Trust, Oxford OX3 9DU, UK

Full list of author information is available at the end of the article

(c) The Author(s) 2021. Open Access This article is licensed under a Creative Commons Attribution 4.0 International License, which permits use, sharing, adaptation, distribution and reproduction in any medium or format, as long as you give appropriate credit to the original author(s) and the source, provide a link to the Creative Commons licence, and indicate if changes were made. The images or other third party material in this article are included in the article's Creative Commons licence, unless indicated otherwise in a credit line to the material. If material is not included in the article's Creative Commons licence and your intended use is not permitted by statutory regulation or exceeds the permitted use, you will need to obtain permission directly from the copyright holder. To view a copy of this licence, visit http://creativecommons.org/licenses/by/4.0/. The Creative Commons Public Domain Dedication waiver (http://creativeco mmons.org/publicdomain/zero/1.0/) applies to the data made available in this article, unless otherwise stated in a credit line to the data. 
identifiers and the tracking of other research outputs such as clinical innovations and patents would allow a more detailed understanding of large research enterprises such as NIHR BRCs in the UK.

Keywords: Research Institutions, Translational Research Organisations, Research productivity, Research outputs, Collaborative research, Author networks

\section{Background}

Translational science requires continuous research and development $(R \& D)$ for advances in scientific understanding to lead to improvements in human health. Therefore, sustainable funding models and streams are critical to support such translational science research [1]. Evidence shows that investment in large research programmes and infrastructure and the inclusion of multi-disciplinary academic and industrial partners [2] are associated with development of new products and processes, high productivity in research outputs such as research publications and patents, and commercialisation and transfer of knowledge and technology [3, 4]. The investment in R\&D also leads to a variety of payback benefits, which include knowledge, research, health, political, administrative and broader economic benefits $[5,6]$.

In addition, translational research provides greater opportunities for multidisciplinary research collaboration which has important implications for scientists, research networks, research partners as well as research policy and outcomes [7]. In the United Kingdom (UK), translational biomedical research involves research collaboration between universities and hospitals and these collaborations become complex because of the research collaborators' diverse structures, procedures and work settings and more importantly due to the complex nature of patients, clinical practice and healthcare delivery $[8,9]$.

Evaluation of these complex translational research collaborations between universities, hospitals and industry as well as between academics and clinicians from diverse disciplines is important [10] but an arduous task. However, evaluation of translational research is critical for learning, management, accountability [11] and assessing the impact of research $[12,13]$. In addition, research evaluation can inform development of strategic policy about research and science, formulation of an institutional research strategy and allocation of research funding [14]. Evaluation of research can be undertaken either prior to or after the completion of the research. The former type of research evaluation involves a review of the study protocol by a Research Ethics Committee/Institutional Review Board and approval is necessary for studies involving human participants [15] whereas the post-study evaluation involves the assessment of the outputs and impacts of the research and this type of research evaluation is helpful in assessing the performance of research projects and programmes, research centres and institutions, and individual research groups [16].

Several indicators have also been developed for evaluating different types of research benefits and impacts. For example, indicators of knowledge production include number of publications, citation rates and journal impact factors [17], whereas co-authorship and co-citation networks are indicators of research capacity building, which can be assessed by bibliometric and case studies [18]. Publications (number and their impact), collaborations (international, regional and national), multidisciplinarity and patents are considered as tier one (direct) impacts of research [19]. The number of citations and highly cited publications are indicators of research quality and these are used for evaluating research and its trends [18].

Research evaluation is a periodic exercise in some countries such as the UK where the Research Evaluation Framework (REF) [20] is an important research evaluation activity that involves assessment of publicly funded research undertaken in higher education institutions (HEI). The precursor of the REF, the Research Selectivity Exercise started in 1986 [16] and it was renamed as Research Assessment Exercise (RAE), which was undertaken in 1992, 1996, 2001 and 2008 [21, 22]. In December 2014, the RAE was replaced with the REF, which assessed the quality of research (QoR) in the national HEIs [20]. In the UK, three REF evaluations have been completed to date and the fourth REF is currently underway [23]. Although REF involves assessment of different elements, research outputs such as publications and the impact of research are the key elements [24]. Based on the findings of the REF, the UK Government funding will be allocated to the HEIs [24].

\section{Introduction of the study setting}

The role of the National Institute for Health Research (NIHR) is to improve the health and welfare of the nation through research [25]. To this end, in 2007, the NIHR established five Biomedical Research Centres (BRCs), where NHS Foundation Trusts work in partnership with the Universities [26]. One of these first five (of 20 to date) BRCs was the NIHR Oxford BRC, a research partnership between the University of Oxford and the Oxford 
University Hospitals NHS Foundation Trust [26]. The NIHR Oxford BRC was originally funded through a competitively awarded grant of $£ 57$ million for 5 years from April 2007 to March 2012 [1]. In the second funding round, the BRC received $£ 95.5$ million (68\% higher than the first funding award) for another 5 year period from April 2012 to March 2017 and in the third competitive funding award, the BRC was successful in getting $£ 114$ million for the period starting from April 2017 to March 2022 [1], which has been extended at least until November 2022 due to the COVID-19 pandemic.

NIHR funding can have direct and indirect routes to better healthcare as well as more general benefits to local and national economy. Measuring effectiveness is important as funding for both research and treatment are finite and there is a need to prioritise spending [27]. In addition, understanding of the barriers and gaps in the pathways translating original research to health benefits requires evaluation of research outputs and metrics, which is also important for self-assessment and correction of measurements [28]. A conventional method of evaluating translational research involves assessment of academic outputs including research publications and citations, which could be better evaluated by bibliometric methods and indicators [29], research network analysis and visualization technologies [30]. With this in mind, we set about gathering the academic research outputs of our second BRC funding cycle covering the period 2012-2017 during which the BRC comprised 14 research themes and six working groups (Additional file 1: Box S1). The research themes were bigger research groups that were established for the first 5-year funding period of the BRC while the working groups were established as newer research 'start ups' for the second 5-year funding period and in some cases expected to be upgraded as research themes in the next BRCs, as part of the BRC's future strategy.

\section{Methods}

\section{Study objective}

The objective of this study was to evaluate translational research productivity, strength and diversity of research collaboration networks and impact of research supported by the NIHR Oxford BRC during its second 5-year period from April 2012 to March 2017.

\section{Outcome measures}

This study included three main outcome measures i.e. research productivity measured by research publications $[17,31]$, research collaboration mapped through co-authorship networks [32, 33], and the quality and impact of research gauged from publication citations $[34,35]$.

\section{Data}

The main data included publications that were defined as those that were reported to the NIHR as the output of the NIHR Oxford BRC between 1st April 2012 and 31st March 2017, which was the second 5-year funding period for the BRC. Individual papers were identified by staff involved in research facilitation within the BRC and from Bodleian Healthcare Libraries in the University of Oxford. Inclusion criteria supplied by NIHR for publications and stipulate amongst other things that "the work was funded/supported by the NIHR funding" [36].

\section{Locating digital object identifiers (DOIs) and metadata}

Initially, we cleaned the publications records and then each research article was matched with its digital object identifier (DOI), which is a unique identifier that makes obtaining further information such as citation data possible. As DOIs were available for only a fraction of the publications when first recorded, a first step was to use the title field to question the Crossref API (https://api. Crossref.org). Where the original record was partial or unmatched after this process, the Crossref text query tool for matching references (https://apps.Crossref.org/simpl eTextQuery) was used alongside manual searches of the bibliographic databases i.e., PubMed and EuroPMC and finally further internet searches where required. This process produced a single DOI for each of the publications in the original list (where one existed).

\section{Using digital object identifiers to acquire citation data}

The unique identifiers for each publication (DOIs) were used to obtain current citation counts for each article from the Crossref (via the REST API, http://api.Crossref. org) and from Dimensions.ai metrics API (Dimensions is an inter-linked research information system provided by Digital Science (https://www.dimensions.ai). In a further attempt to establish a baseline for these newer metrics, Dimensions metrics API data was also collected for 500 randomly selected DOIs from Crossref.

In the citation analysis, different time periods, known as citation windows, are used such as total citations over 2 years, 5 years [37] or 10 years [38]. In the current study, we used a mix of citation window covering time periods ranging between 8 and 3 years for publications published between 2012 and 2017 respectively.

\section{Author numbers per publication}

The total number of authors was calculated, using the author field of the Crossref citations. Many research publications also reference research consortia, often in place of individual authors where research consortia were described, and a full list of authors was apparent in the article or appendices. 


\section{Production of co-authorship networks}

All the DOIs were used to obtain lists of authors for each publication, as a whole, or equal-sized lists of DOIs representing the start, middle, and end of the funding period being studied. These lists of DOIs were used to construct a series of author co-authorship (association) networks, using Python scripts (Notebook 'D') (please see, Additional file 1: Figure S1). Where individual authors were associated with more than one research group, all associations were recorded, with the most prevalent used as the primary group (or type of group) for the author. Networks were also explored in the program VOSviewer [39] (version 1.6.11, https://www.vosvi ewer.com/) for comparison (included in analysis extra notebooks).

The resulting network files (.gml or .GEXF) were exported for visualization analysis in the Gephi (version 0.9.2, https://gephi.org/) [40]. All networks were analysed within Gephi to obtain measure of complexity (nodes and edges) and connectivity (average path length) and to filter networks for final figures. Where metrics were also calculated in the Python Networkx library the results were identical to those from Gephi.

\section{General methodology-data availability and tools used}

The final analysis for the metrics was run on the 27 th January 2021 (when data was obtained from the respective APIs). Python (Jupyter) notebooks are available describing the entire analysis from the original curated list of publications, through to the lists of DOIs used to generate the author networks. Majority of the analysis was run from these notebooks, except for a final manual check of the available titles and identifiers. The analyses make use of a number of packages from the PyData ecosystem, including Jupyter [41], IPython [42], Pandas [43, 44], Numpy [45], Scipy [46], Holoviz libraries (Bokeh [47], Hvplot, Holoviews [48], Panel, Networkx [46], Requests, FuzzyWuzzy and Habanero for the Crossref API).

We created different notebooks for the analysis (Additional file 1: Figure S1). All data are available in GitHub Repository [49] and a snapshot of code and data used (including network files) have been uploaded on the Zenodo Repository [50].

\section{Results}

\section{Research publications}

A total of 2377 publications were reported to the NIHR as the academic research output for the period from 1st April 2012 to 31st March 2017 (Table 1). Matching this list to Crossref records by title and DOI (or by full record), followed by a manual check of outstanding references allowed DOIs to be identified for all but 12 of these publications. Those publications still lacking DOIs after this process were commentaries, lecture notes, or book chapters without DOIs assigned. In a couple of cases DOIs were broken or unregistered (these were reported). This left 2365 of 2377 with valid DOIs (99.5\%) (Table 1).

\section{Research collaboration and authorship}

Two thousand one hundred and thirty-five publications were reported by all 14 Research Themes (established groups prior to this funding period) and 219 publications by six Working Groups (Fig. 1). Additionally, 23 publications were reported by 'Other' research groups i.e., Ethics group and Health Economic group, which were formal groups of the BRC. Less than $5 \%$ of the publications were reported by more than one research group.

Author details were available via Crossref for 2359 of the 2377 original listed publications $(99.75 \%$ of the 2365 with DOIs). Although some publications were the work of a single author, the level of collaborative work within the NIHR Oxford BRC was notable, as were the extremes. The overall median authors number was 9, while the greatest number of authors was 2467 [51]. The mean authors number of these publications was 18.03 (SD 3.63), but it was clear that this figure was inflated by outliers, a few mega-author papers [17, 52]. In fact, 57 publications had more than 100 listed authors. The average number of authors varied greatly within and between Research Themes and Working Groups (Fig. 2). Research Themes had a median of nine authors in comparison to a median five authors for the Working Groups (Fig. 2).

\section{Co-authorship networks}

One possible way of looking at how a large research group functions is to examine connections between researchers as a series of edges (co-authors) between nodes (individual authors). Considering the 2365 publications

Table 1 Total publications, unique digital object identifiers and citations

\begin{tabular}{lll}
\hline & Count (n) \\
\hline Total publications in manually collated list & 2377 \\
Digital object identifiers (DOIs) found with Crossref API & 2365 \\
Data from Dimensions.ai metrics API & 2364 \\
Citations data in Crossref & 2361 \\
\hline
\end{tabular}




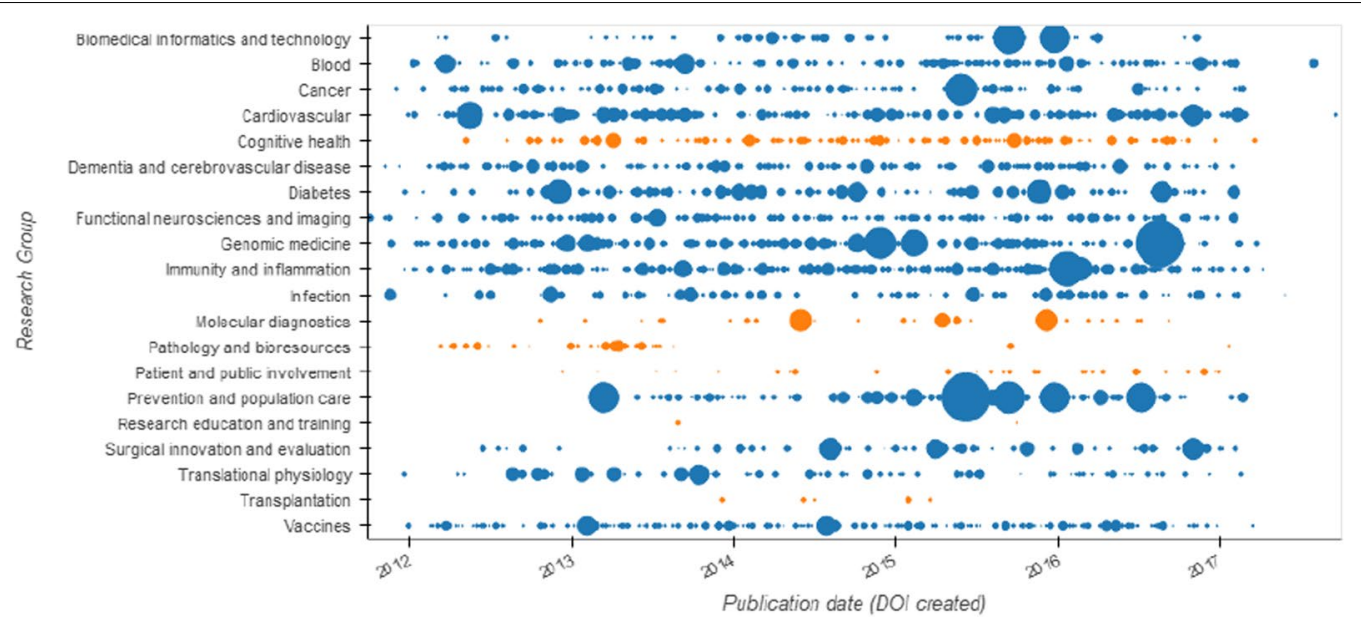

Fig. 1 NIHR Oxford BRC publications between April 2012 and March 2017 divided by research themes (blue) and working groups (orange). Each node is a publication with a DOI, and the size of the node relates to the field citation ratio for that publication as of 27th January 2021

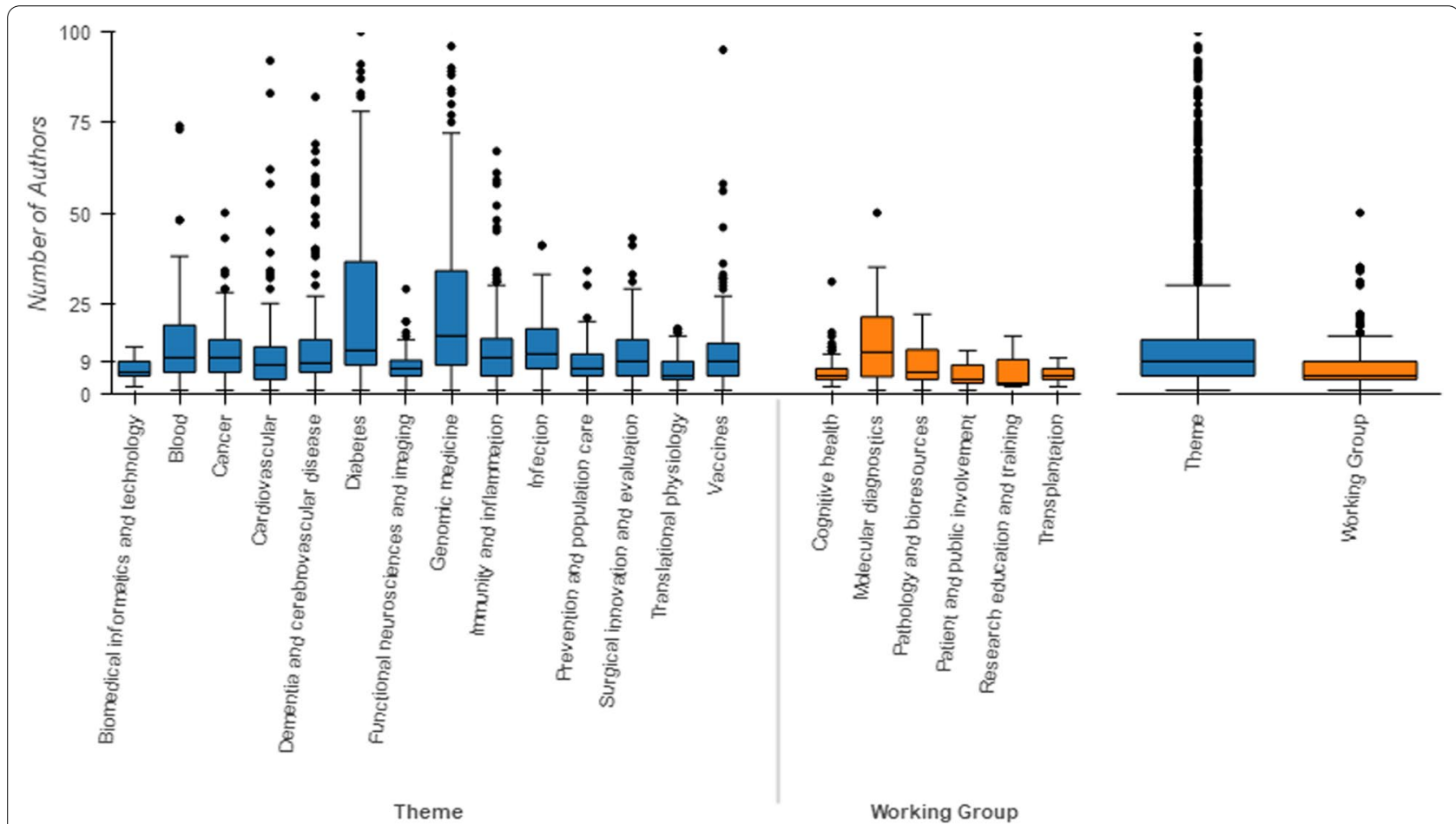

Fig. 2 Authors per publication by research themes and working groups. Boxplot for numbers of authors (box = median and quartiles, whiskers $=1.5 \times$ Interquartile range)

examined and the high average authorship, it was not surprising that the derived association network of authors was substantial. Without disambiguation beyond reducing names to initials, the co-authorship networks for the analysed publications comprised more than 20 thousand nodes (individual authors) and close to 4.3 million edges (co-authors) (Table 2). The entire co-authorship network of the BRC during the study period was a dense network having density of 0.021 (the number of edges as a proportion of the maximum possible) (Table 2).

Dividing the total publications into 3 stages, the derived co-authorship networks from start, middle, and end of the second 5-year funding period of the BRC indicate a further strengthening of an existing network 
Table 2 Attributes of co-authorship networks built from publications of the NIHR Oxford BRC (April 2012-March 2017)

\begin{tabular}{|c|c|c|c|c|c|c|c|c|c|}
\hline \multirow[t]{2}{*}{ Publication period } & \multicolumn{5}{|c|}{ Measures of co-authorship networks ${ }^{a}$} & \multirow[t]{2}{*}{ Oxford nodes $^{b}$} & \multicolumn{3}{|c|}{ Authors per DOI } \\
\hline & Nodes & Edges & Density & $\begin{array}{l}\text { Average path } \\
\text { length }\end{array}$ & $\begin{array}{l}\text { Network } \\
\text { diameter }\end{array}$ & & Mean & Median & Max \\
\hline Start & 6,684 & 288,614 & 0.013 & 3.880 & 11 & 868 & 14.42 & 8 & $322^{c}$ \\
\hline Mid & 8,697 & 786,972 & 0.021 & 3.539 & 10 & 1,006 & 18.27 & 8 & 679 \\
\hline End & 10,913 & $3,534,201$ & 0.059 & 3.032 & 10 & 1,068 & 21.67 & 9 & 2,467 \\
\hline All publications & 20,225 & $4,292,252$ & 0.021 & 3.076 & 7 & 1,606 & 18.03 & 9 & \\
\hline
\end{tabular}

${ }^{a}$ Network measures derived from Gephi after summation of edge weights on import

${ }^{b}$ Oxford nodes are defined as those in which the word Oxford was found within the author's primary affiliation

${ }^{c}$ Maximum without resolving all consortia and groups in publications (start section includes a known consortium of over 1200 individuals)

over time, with increasing density of connections overall (proportion of possible connections) and a decrease in the average path length (shortest route between any two individuals in the network (Table 2). Although there was a slight increase in the median authorship per publication, much of the substantial increase in authors in the last third of the second 5-year funding period of the BRC appeared to be due to occasional publications with very large author lists up to 2467 (Table 2).

Another measure of increasing collaboration with the research network is decreasing average path length over time (average number of connections needed to join any two authors). Visualizing the author network revealed tight integration with widespread interaction between research themes and working groups. The working groups, the newer research groups that were launched during the second 5-year funding period of the NIHR Oxford BRC, clearly derived from existing research networks in most cases and all were closely linked to ongoing work throughout the BRC (Fig. 3).

\section{Research consortia}

Of the authors listed in analysed publication records (20,229 in total from Crossref), a total of 120 contained the term 'Consortium' with other entries also indicating multiple contributors, such as 'Group' [40], 'Committee' [6], and 'Team' [2]. In many cases, the size of these groupings was not stated, therefore prevented the actual authorship of the paper to be counted. It was

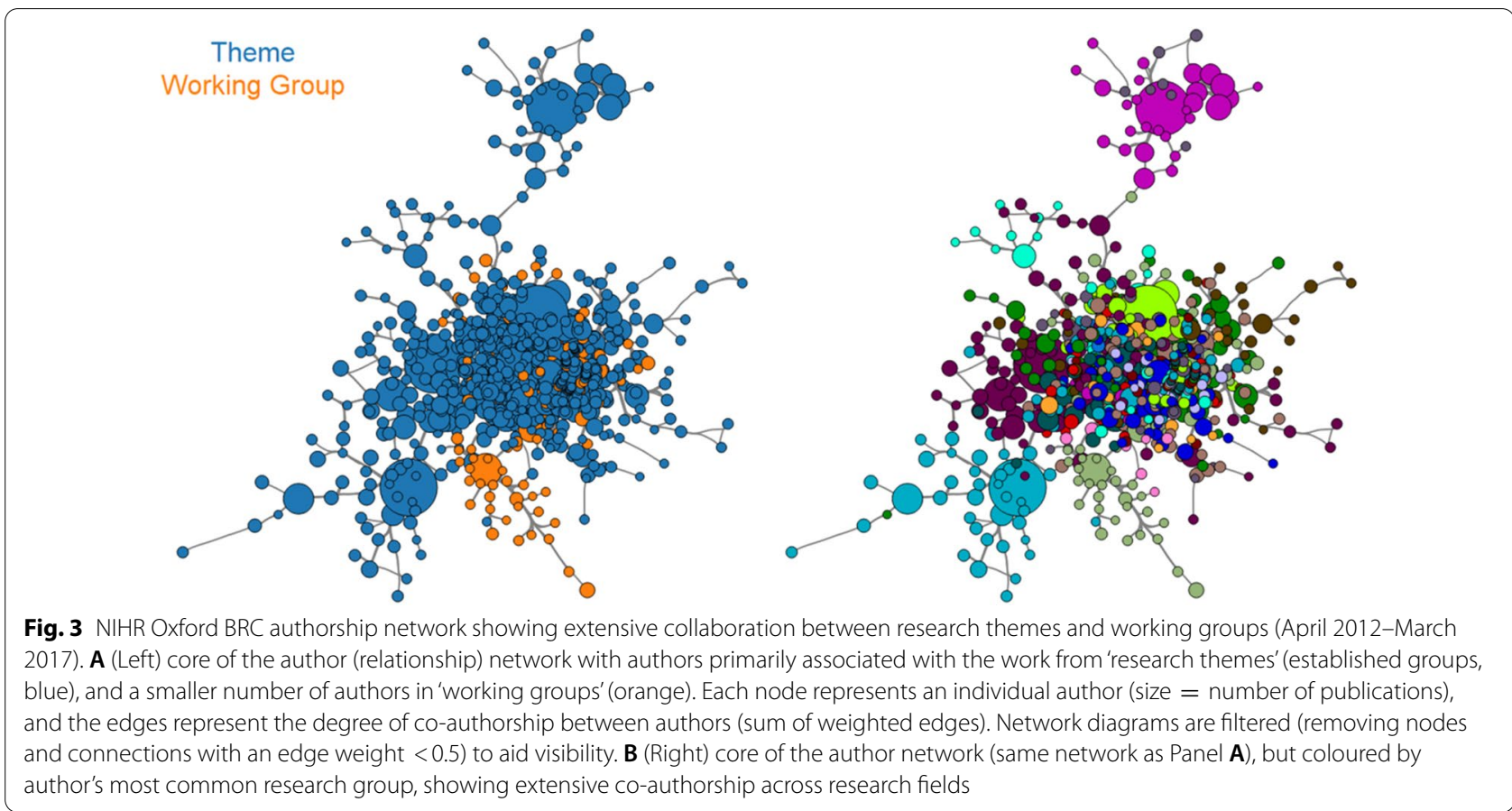


notable that a brief examination of papers reporting as a group, there were up to 1238 additional contributors within a publication that were represented as a single 'author' [53].

\section{Publication citations and citation ratios}

As of 27th January 2021, the NIHR Oxford BRC articles (published between 1 April 2012 and 31 March 2017) with DOIs were cited more than 155,000 times. The most cited article was cited over 6000 times since its publication in 2016 (Table 3).

Perhaps of more value is the availability of citation ratios, where publications are compared to others of the same research field and age. The geometric mean (to avoid influence of outliers, [54]) FCR for all analysed publications was 7.12, which indicated more than 7-times the number of citations the average paper in the same research area and with same age received (Fig. 4). The publications (as assessed by citation ratios) had a similar impact across all research areas. There was a substantial variation in the sizes of Research Themes and Working Groups in different fields, but despite this the mean FCRs for all research themes and working groups were above 1 (Fig. 4).

\section{Discussion}

In this study, we retrospectively evaluated different bibliometric measures for the NIHR Oxford BRC for its second 5-year funding period from 1st April 2012 to 31st March 2017. The studied three metrics i.e., research publications as a measure of research productivity [17, 31], co-authorship networks as a measure of research collaboration [32,33], and publication citations as an indicator of the quality and impact of research [34, 35]. These bibliometrics are important in measuring and assessing the success of a large-scale research effort and have been used for the evaluation of research and impact in the context of biomedical research [55] including translational research $[35,56]$.

Table 3 Citations and citation ratios of NIHR Oxford BRC papers published from April 2012 to March 2017

\begin{tabular}{|c|c|}
\hline & Count $^{a}$ \\
\hline Total publications with digital object identifiers (of total publications) & 2365 (of 2377) \\
\hline Total citations, Crossref API (n) & $155,699(n=2361)$ \\
\hline Total citations, Dimensions.ai metrics API (n) & $173,995(n=2364)$ \\
\hline Equivalent h-index (current n publications with at least n citations) & 166; 178 (Crossref; Dimensions.ai) \\
\hline Average field citation ratio from Dimensions.ai metrics API (n) & 7.12 geometric mean, 6.75 median $(n=2259)$ \\
\hline Average relative citation ratio from Dimensions.ai metrics API (n) & 1.83 geometric mean, 1.50 median $(n=2300)$ \\
\hline
\end{tabular}

${ }^{\text {a }}$ Overall counts for NIHR Oxford BRC publications and comparison of rates of citation with similar research publications (from publication to 27th January 2021)

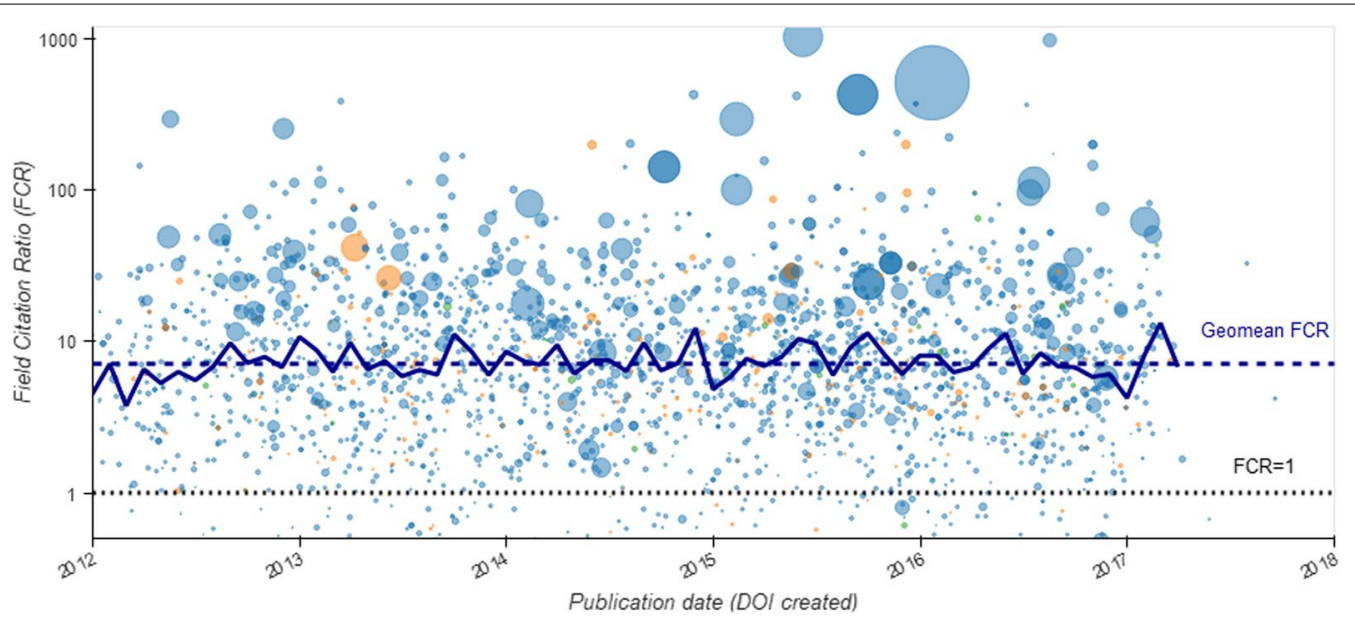

Fig. 4 Field Citation Ratios for publications of the NIHR Oxford BRC over time coloured by research group (April 2012 - March 2017). Each node is a publication with a DOI, and the size of the node relates to the number of authors listed for the publication. Dashed blue line represents the geometric mean (g mean) FCR 7.12 for the entire publications of the NIHR Oxford BRC published during the study period and the dotted black line represents an $F C R=1$ (citation rate for a similar publication of the same age). Solid blue line is the monthly $g$ mean of the FCR, showing a very consistent output during the funding period (1st April 2012 - 31st March 2017) 
Our findings showed that the research productivity output of the NIHR Oxford BRC during the study period was consistently producing about 40 publications a month, every month for 5 years from April 2012 to March 2017. This linear publication rate masks differences in size and publication rates between research groups. One notable difference was the ten-fold higher rate of reported publications from the research themes, which were established research groups of the BRC prior to the study period, when compared to the working groups that were newer and established at the start of the analysed period. Regardless of research area, the citation rates on average for each research group indicate higher impact of the work compared to similar publications (all citation ratios were above 1) (Fig. 1).

Although an individual researcher level is the most common unit of analysis in studies on research productivity [57], in this study we analysed research productivity through publications at the environment level i.e., at the level of our BRC, which provides research facilities and funds as well as recognition, which are crucial factors in promoting research productivity [57] and fostering research collaboration that are positively correlated with each other [58]. Though there is a strong correlation between the quantity and impact of research such as the number of publications and number of citations respectively [34, 35], this is an area that deserves more attention [59] and maintaining a balance between the quantity and the quality of research is crucial [60]. It is also notable that evaluating research(er's) productivity, quality and impact is not an easy task [61] and putting targets on research productivity, measured as number of publications, is a much debated and controversial issue [62].

We found a highly integrated nature of co-authorship network showing highly collaborative research working of the BRC and closer and stronger associations between researchers, which were evident from the average path length [63] and density of the network [64] as shown in Table 2. Whilst there was a perception that the BRC was important in building a robust co-authorship network, it becomes clear from this data that the network was already at least partially established at the start of second 5 -year funding period of the BRC. However, there were further increases in the co-authorship network's density and stability during the study period. It also seems that newer working groups developed with close associations to existing research themes, rather than in isolation. A true understanding of the developing research network will come from further study of the first and ongoing third 5-year funding periods of the NIHR Oxford BRC and it would be best compared to similar clinical research networks and centres, such as other NIHR BRCs in the country.
The large numbers of consortia and groups named with authorship of analysed publications is also interesting, in part because these may represent an important transition from exploratory research to an agreed vision about a route to improving healthcare and accompanying organisation and governance. Some studies have explored what factors help consortia succeed, such as scientists who are rewarded are productive and vice versa [65]. The development of a research consortium could potentially be seen as an acknowledged need for data standards and a shared voice in research and policy. The process of establishing a research consortium can be an important step in the translation of basic research to direct health benefits. However, the reporting of consortia is variable, particularly how membership relates to authorship on publications. This is another area where variations in publishing guidelines and reporting further complicates conclusion. In addition, it may be interesting to focus in the future on if there are measures (e.g., geographical diversity) that change during a large research endeavour such as the NIHR Oxford BRC.

Another important issue in relation to the performance and impact could be tackling the gender gap in scientific authorship, which could be reduced by promoting and providing fair and equitable opportunities especially to female scientists, early career researchers (both male and female) and researchers of ethnic minority background. Our earlier study on the gender equity in the authorship of scientific publications produced by researchers affiliated with the NIHR Oxford BRC revealed that although the overall proportion of female authors was lower than male authors, there were significant increasing trends of female first, last and corresponding authors and the proportions of male and female last authors were similar to their respective proportions as principal investigators in the BRC [66].

Publications analysed in this study were published in more than 700 different journals from one hundred scientific publishers with a wide variation in journal styles and formats that provide many options for how scientific progress is reported, allowing different fields and types of work to find suitable voice. However, this diversity in editorial rules and practices means substantial variability in bibliometric data. The publication process (often months of cyclical peer review and often rejection) makes it very difficult to accurately link work to a specific date. In addition, there are a multitude of minor editing decisions that can particularly affect consistency of data, from stylistic changes to lists of authors to the trimming of acknowledgements that sees funding statements removed or changed. These changes all increase the variability in the data. In recent years many publishers have come together to support efforts such as Crossref [67] to help 
the interchange of data within the industry improving the standards of such aggregated data.

It is also important to acknowledge to substantial changes taking place in the world of scientific publishing right now, with 'Plan $S$ ' an example of funders driving change to make knowledge more accessible to all [68]. Clear transparency and inclusive attempts to allow the interchange of data, as represented by efforts like Crossref, are essential and welcome.

Tracking the citation metrics of individual papers is now routine for various purposes such as checking the quality, significance and impact of research [37]. In addition, many other ways of establishing visibility and interest (or 'impact') being explored. However, the impact of international co-publication is amplified by self-citations, which might be unavoidable [69] but may distort or create bias in the impact of the publication or research. Hence self-citations must be corrected to gauge the actual and unbiased impact [69]. Citation metrics with more context and granularity are needed and it is useful when these are developed without restricting access. There has been progress in this area too [70]. In the hunt for better measurements of knowledge gathering and dissemination, it is essential to allow examination of the underlying data whenever possible.

There are many useful outputs from the academic research process, yet few of these are reported. This may be even truer in medical research, where valuable datasets, clinical checklists, and policy documents can often derive from research. Efforts continue to ensure the scheme for the REF measures the full value of academic study. However, the most recent REF exercise in 2014 saw $97 \%$ of the items offered up for assessment were papers or book chapters (https://hidden-ref.org/) [71]. This lack of visibility for many valuable products of research may make it even more difficult to track the steps that were essential in successful health and welfare breakthroughs.

\section{Research policy implications}

At the heart of efforts to improve accuracy in bibliometrics is widening the availability and usage of Persistent Identifiers (PIDs). Such identifiers for publications, datasets, as well as individual researchers (ORCID [72]) and research organisations/institutions (GRID [73] and ROR [74]) will allow insights at many levels and are required to move beyond blunt journal-level measurements. This is a rapidly moving field and one where great efforts are being made to move to PIDs for many aspects of research, as much to capture the relationships between different data types as to count each one $[75,76]$. Unique identifiers are thus essential for tracking anything accurately, be that a researcher, dataset, or publication. The uptake of such PIDs will be partly dependent on their visibility and availability, but also on researchers understanding how these will improve accuracy of measurement. Widespread use of PIDs, not just for publications, but also for researchers and data, must become central to publishing. Defining and adopting standard identifiers for other stages on the paths of translation to healthcare benefits [1] will also be needed.

\section{Strengths and limitations}

We have used this study to self-assess translational research productivity and discuss current issues and the future areas of interest in the field. We have used freely available tools and data sources in this first exploration, to provide NIHR Oxford BRC members and collaborators as much data and code as possible. This process is important considering the uncertainty and limitations in some of the data as well as sources of variation in the data analysed.

\section{Conclusion}

The substantial output of researchers supported by the NIHR Oxford BRC during its second 5-year funding period produced substantial number of research publications, which were generated by a large and complex network of translational researchers working in complex structures and consortia, which shows success across the BRC during the period of analysis. Further research involving continued improvements in and uptake of PIDs, open data and the tracking of more other research outputs such as clinical innovations and patents should give a better understanding of large research enterprises such as NIHR BRCs in the UK. In addition, variations in reporting of authorship and the lack of PIDs must be acknowledged.

\begin{abstract}
Abbreviations
BRC: Biomedical Research Centre; DOI: Digital object identifier; FCR: Field citation ratio: a citation-based measure of scientific influence of one or more articles; FCR Mean: The average field citation ratio_-indicates the relative citation performance of an article, when compared to similarly aged articles in the same fields of research category; FoR: Fields of research - a classification system covering all areas of research from Arts and Humanities to Science and Engineering; NIHR: National Institute of Health Research; PIDs: Persistent identifiers; REF: Research evaluation framework; RAE: Research assessment exercise; R\&D: Research and development; RCR: Relative citation ratio; QoR: Quality of research; UK: United Kingdom; WG: Working group.
\end{abstract}

\section{Supplementary Information}

The online version contains supplementary material available at https://doi. org/10.1186/s12967-021-03149-x.

Additional file 1: Box S1. Research themes and working groups. Figure S1. Notebooks for analysis. 


\section{Acknowledgements}

The authors thank Owen Coxall and the Bodleian Healthcare Libraries team at the University of Oxford, as well as the BRC managers, theme liaisons and administrators from BRC2 for their efforts to collect and collate publications each year of the project. We would also like to thank Joe Wass at Crossref for useful discussion.

\section{Authors' contributions}

VK: conceptualization; funding acquisition; investigation; methodology; project administration; data collection, resources; supervision; writing - review and editing. LAB: data curation; formal analysis; methodology; software; visualization; validation; writing — original draft. HMCS and KMC: conceptualization; funding acquisition; resources; writing - review and editing. SGSS: conceptualization; data curation; methodology; validation; writing —original draft, review, editing and finalising. All authors read and approved the final manuscript.

\section{Funding}

This work was funded/supported by the National Institute for Health Research (NIHR) Oxford Biomedical Research Centre (Research Grant Number IS-BRC-1215-20008). The views expressed are those of the author(s) and not necessarily those of the National Health Service, the NIHR, or the Department of Health.

\section{Availability of data and materials}

All data are available in GitHub Repository [49], and a snapshot of code and data used (including network files) have been uploaded on the Zenodo Repository [50].

\section{Declarations}

\section{Ethics approval and consent to participate}

Not applicable.

\section{Consent for publication}

Not applicable.

\section{Competing interests}

VK is the Chief Operating Officer and HMCS is the Director of the NIHR Oxford BRC. KMC is R\&D Director of the OUH and was the Director of the NIHR Oxford BRC from 2012 to 2017. SGSS is Senior Research Fellow at the NIHR Oxford $B R C$

\section{Author details}

'NIHR Oxford Biomedical Research Centre, John Radcliffe Hospital, Oxford University Hospitals NHS Foundation Trust, Oxford OX3 9DU, UK. ${ }^{2}$ Nuffield Department of Primary Care Health Sciences, University of Oxford, Radcliffe Primary Care Building, Woodstock Road, Oxford OX2 6GG, UK. ${ }^{3}$ Research Support Team, IT Services, University of Oxford, Oxford OX1 2JD, UK. ${ }^{4}$ Nuffield Department of Clinical Neurosciences, John Radcliffe Hospital, University of Oxford, Oxford OX3 9DU, UK. ${ }^{5}$ Nuffield Department of Medicine, The Jenner Institute, University of Oxford, Old Road Campus, Oxford OX3 7BN, UK. ${ }^{6}$ Division of Cardiovascular Medicine, British Heart Foundation (BHF) Centre of Research Excellence, John Radcliffe Hospital, University of Oxford, Oxford OX3 9DU, UK. ${ }^{7}$ Radcliffe Department of Medicine, Medical Sciences Division, John Radcliffe Hospital, University of Oxford, Oxford OX3 9DU, UK.

Received: 4 October 2021 Accepted: 15 November 2021 Published online: 27 November 2021

\section{References}

1. Miyashita S, Katoh S, Anzai T, Sengoku S. Intellectual property management in publicly funded R\&D program and projects: optimizing principalagent relationship through transdisciplinary approach. Sustainability. 2020;12(23):9923

2. D'lppolito B, Miozzo M, Consoli D. Knowledge systematisation, reconfiguration and the organisation of firms and industry: the case of design. Res Policy. 2014;43(8):1334-52.
3. Bodas Freitas IM, Verspagen B. The motivations, organisation and outcomes of university-industry interaction in the Netherlands. Maastricht Economic and Social Research and Training Centre on Innovation and Technology. Maastricht: United Nations University; 2009.

4. Goddard JG, Isabelle M. How do public laboratories collaborate with industry? New survey evidence from France. SSRN. Working Paper No. IMRI 2006/02. Paris, France: Paris Dauphine University; 2006.

5. Buxton M, Hanney S. How can payback from health services research be assessed? J Health Serv Res Policy. 1996;1(1):35-43. https://doi.org/10. 1177/135581969600100107.

6. Hanney S, Greenhalgh T, Blatch-Jones A, Glover M, Raftery J. The impact on healthcare, policy and practice from 36 multi-project research programmes: findings from two reviews. Health Res Policy Syst. 2017;15(1):26-26.

7. D'Ippolito B, Rüling C-C. Research collaboration in Large Scale Research Infrastructures: collaboration types and policy implications. Res Policy. 2019;48(5):1282-96.

8. Munoz DA, Nembhard HB, Kraschnewski JL. Quantifying complexity in translational research: an integrated approach. Int J Health Care Qual Assur. 2014;27(8):760-76. https://doi.org/10.1108/IJHCQA-01-2014-0002.

9. Qidwai W. Translational research and complexity of clinical practice: issues, challenges, and way forward. J Coll Physicians Surg Pak. 2016;26(6):453-4

10. Long JC, Cunningham FC, Carswell P, Braithwaite J. Patterns of collaboration in complex networks: the example of a translational research network. BMC Health Serv Res. 2014;14(1):225. https://doi.org/10.1186/ 1472-6963-14-225.

11. Trochim W, Kane C, Graham MJ, Pincus HA. Evaluating translational research: a process marker model. Clin Transl Sci. 2011;4(3):153-62. https://doi.org/10.1111/j.1752-8062.2011.00291.x.

12. Sung NS, Crowley J, William F, Genel M, Salber P, Sandy L, Sherwood LM, et al. Central challenges facing the national clinical research enterprise. JAMA. 2003;289(10):1278-87. https://doi.org/10.1001/jama.289.10.1278.

13. Kim YH, Levine AD, Nehl EJ, Walsh JP. A bibliometric measure of translational science. Scientometrics. 2020;125(3):2349-82. https://doi.org/10. 1007/s11192-020-03668-2.

14. van Steen J, Eijffinger M. Evaluation practices of scientific research in the Netherlands. Res Eval. 1998;7(2):113-22. https://doi.org/10.1093/rev/7.2. 113.

15. Amdur RJ, Biddle C. Institutional review board approval and publication of human research results. JAMA. 1997;277(11):909-14.

16. Geuna A, Martin BR. University research evaluation and funding: an international comparison. Minerva. 2003;41(4):277-304. https://doi.org/ 10.1023/B:MINE.0000005155.70870.bd

17. Fire M, Guestrin C. Over-optimization of academic publishing metrics: observing Goodhart's Law in action. GigaScience. 2019; 8(6): giz053. https://doi.org/10.1093/gigascience/giz053.

18. Frank C, Battista R, Butler L, Buxton M, Chappell N, Davies SC, et al. Making an impact, a preferred framework and indicators to measure returns on investment in health research. Ottawa: Canadian Academy of Health Sciences (CAHS); 2009.

19. Beaudet A. CAHS forum summary: the return on investments in health research: defining the best metrics. Montreal: Canadian Academy of Health Sciences; 2007.

20. Research Excellence Framework. About the REF. REF2014 research excellence framework. 2014. https://www.ref.ac.uk/2014/. Accessed 25 Nov 2020.

21. Parliament UK. Research assessment exercise: a re-assessment. Eleventh report of session 2003-04. Report No.: HC586. London: House of Commons; 2004.

22. Stern N. Building on success and learning from experience. An independent review of the research excellence framework. London: Department for Business, Energy \& Industrial Strategy; 2016

23. Research Excellence Framework. About the REF. REF 2021 research excellence framework. 2020. https://www.ref.ac.uk/about/. Accessed 25 Nov 2020.

24. Research Excellence Framework. Guidance on providing testimonies for REF 2021 impact case studies. 2020. https://www.ref.ac.uk/guidance/ additional-guidance/. Accessed 25 Nov 2020. 
25. National Institute for Health Research (NIHR). Our mission and core workstreams. Department of Health and Social Care, United Kingdom. 2020. https://www.nihr.ac.uk/about-us/our-mission/our-mission-and-strategicworkstreams.htm. Accessed 24 Feb 2021.

26. NIHR Oxford Biomedical Research Centre. About the NIHR Oxford Biomedical Research Centre. 2020. https://oxfordbrc.nihr.ac.uk/about-usintro/. Accessed 4 Sep 2020

27. Greenhalgh T, Ovseiko PV, Fahy N, Shaw S, Kerr P, Rushforth AD, et al. Maximising value from a United Kingdom Biomedical Research Centre: study protocol. Health Res Policy Syst. 2017;15(1):70. https://doi.org/10.1186/ s12961-017-0237-1.

28. Hicks D, Wouters P, Waltman L, De Rijcke S, Rafols I. Bibliometrics: the Leiden Manifesto for research metrics. Nature. 2015;520(7548):429-31.

29. Franceschini F, Maisano D. Structured evaluation of the scientific output of academic research groups by recent h-based indicators. J Informetr. 2011;5(1):64-74

30. Yao Q, Lyu P-H, Ma F-C, Yao L, Zhang S-J. Global informetric perspective studies on translational medical research. BMC Med Inform Decis Mak. 2013;13(1):77. https://doi.org/10.1186/1472-6947-13-77.

31. Koya K, Chowdhury G. Metric-based vs peer-reviewed evaluation of a research output: lesson learnt from UK's national research assessment exercise. PLoS ONE. 2017;12(7):e0179722-e0179722.

32. Chen K, Zhang Y, Zhu G, Mu R. Do research institutes benefit from their network positions in research collaboration networks with industries or/and universities? Technovation. 2020;94-95:102002.

33. Marchand GC, Hilpert JC, Bragg KM, Cummings J. Network-based assessment of collaborative research in neuroscience. Alzheimer's Dement Trans Res Clin Interv. 2018;4:433-43.

34. Ramsden P. Describing and explaining research productivity. High Educ. 1994;28(2):207-26. https://doi.org/10.1007/BF01383729.

35. Schneider M, Kane CM, Rainwater J, Guerrero L, Tong G, Desai SR, et al. Feasibility of common bibliometrics in evaluating translational science. J Clin Transl Sci. 2017;1 (1):45-52.

36. National Institute for Health Research (NIHR). NIHR research outputs and publications guidance. 2020. https://www.nihr.ac.uk/documents/nihr-resea rch-outputs-and-publications-guidance/12250. Accessed 16 Dec 2020.

37. Todeschini R, Baccini A. Handbook of bibliometric indicators: quantitative tools for studying and evaluating research. Weinheim: Wiley; 2016.

38. Huang J, Gates AJ, Sinatra R, Barabási A-L. Historical comparison of gender inequality in scientific careers across countries and disciplines. Proc Natl Acad Sci USA. 2020;117(9):4609.

39. van Eck NJ, Waltman L. Software survey: VOSviewer, a computer program for bibliometric mapping. Scientometrics. 2010;84(2):523-38. https://doi.org/ 10.1007/s11192-009-0146-3.

40. Bastian M, Heymann S, Jacomy M. Gephi: an open source software for exploring and manipulating networks. In: Proceedings of the Third International ICWSM Conference. San Jose: The AAAI Press; 2009. p. 361-2.

41. KluyverT, Ragan-Kelley B, Pérez F, Granger BE, Bussonnier M, Frederic J, et al. Jupyter Notebooks - a publishing format for reproducible computational workflows. In: Loizides F, Schmidt B, editors., et al., Ebook: positioning and power in academic publishing: players, agents and agendas. Amsterdam: IOS Press; 2016. p. 87-90. https://doi.org/10.3233/978-1-61499-649-1-87.

42. Perez F, Granger BE. IPython: a system for interactive scientific computing. Comput Sci Eng. 2007;9(3):21-9.

43. Reback J, McKinney W, den Bossche J, Jbrockmendel A, Augspurger T, Cloud P, et al. Pandas v0.25.3. 10.5281/zenodo.3524604. Zenodo. 2019. https:// github.com/pandas-dev/pandas/tree/v0.25.3. Accessed 24 Dec 2020.

44. McKinney W. Python for data analysis. Sebastopol: O'Reilly; 2013.

45. van der Walt S, Cilbert C, Varoquaux G. The NumPy array: a structure for efficient numerical computation. Comput Sci Eng. 2011;13:22-30.

46. Hagberg AA, Schult DA, Swart PJ. Exploring network structure, dynamics, and function using NetworkX. In: Varoquaux G, Vaught T, Millman J, editors. Proceedings of the 7th Python in Science conference (SciPy 2008). Pasadena: SciPy.org; 2008. p. 11-5.

47. Bokeh Development Team. The Bokeh visualization library. 2018. https:// bokeh.org/. Accessed 12 Mar 2021.

48. Stevens JLR, Rudiger P, Bedner JA. HoloViews: building complex visualizations easily for reproducible science. In: Huff K, Bergstra J, editors. Proceedings of the 14th Python in Science Conference (SciPy 2015). Austin: SciPy. org; 2015. p. 59-66.
49. Kiparoglou V, Brown LA, MCShane H, Channon KM, Shah SGS. Index of notebooks for OxBRC2 bibliometric analysis. San Francisco: GitHub Inc:; 2021. https://github.com/LozRiviera/BRC_Oxford2_bibliometrics/tree/published. Accessed 4 Oct 2021.

50. Brown LA, Kiparoglou V, McShane H, Channon KM, Shah SGS. Data and code for: a large National Institute for Health (NIHR) Biomedical Research Centre Facilitates Cross-Disciplinary and Collaborative Research Outputs: a bibliometric analysis. 2021. Zenodo. https://doi.org/10.5281/zenodo.52112 98.

51. Klionsky DJ, Abdelmohsen K, Abe A, Abedin MJ, Abeliovich H, Arozena AA, et al. Guidelines for the use and interpretation of assays for monitoring autophagy (3rd edition). Autophagy. 2016;12(1):1-222. https://doi.org/10. 1080/15548627.2015.1100356.

52. Regalado A. Multiauthor papers on the rise. Science. 1995;268(5207):25.

53. The IST-3 Collaborative Group. The benefits and harms of intravenous thrombolysis with recombinant tissue plasminogen activator within $6 \mathrm{~h}$ of acute ischaemic stroke (the third international stroke trial [IST-3]): a randomised controlled trial. Lancet. 2012;379(9834):2352-63. https://doi.org/ 10.1016/S0140-6736(12)60768-5.

54. Thelwall M, Fairclough R. Geometric journal impact factors correcting for individual highly cited articles. J Informetr. 2015;9(2):263-72.

55. Rosas SR, Kagan JM, Schouten JT, Slack PA, Trochim WMK. Evaluating research and impact: a bibliometric analysis of research by the NIH/NIAID HIV/AIDS clinical trials networks. PLoS ONE. 2011;6(3):e17428. https://doi. org/10.1371/journal.pone.0017428.

56. Yu F, Van AA, Patel T, Mani N, Carnegie A, Corbie-Smith GM, et al. Bibliometrics approach to evaluating the research impact of CTSAs: a pilot study. J Clin Transl Sci. 2020;4(4):336-44.

57. Fox MF. Publication productivity among scientists: a critical review. Soc Stud Sci. 1983;13(2):285-305. https://doi.org/10.1177/030631283013002005.

58. Abramo G, D'Angelo CA, Di Costa F. Research collaboration and productivity: is there correlation? High Educ. 2009;57(2):155-71. https://doi.org/10. 1007/s10734-008-9139-z.

59. VanderZanden A, Langlois EV, Ghaffar A, Bitton A, Fifield J, Hirschhorn LR. It takes a community: a landscape analysis of global health research consortia. BMJ Glob Health. 2019;4(Suppl 8):e001450.

60. Stallings J, Vance E, Yang J, Vannier MW, Liang J, Pang L, et al. Determining scientific impact using a collaboration index. Proc Natl Acad Sci USA. 2013;110(24):9680.

61. Sahel J-A. Quality versus quantity: assessing individual research performance. Sci Transl Med. 2011;3(84):84cm13.

62. Siegel D, Baveye P, Sills J. Battling the paper glut. Science. 2010;329(5998):1466.

63. Uddin S, Hossain L, Rasmussen K. Network effects on scientific collaborations. PLOS ONE. 2013;8(2):e57546-e57546.

64. van Leeuwen T, Hoorens S, Grant J. Using bibliometrics to support the procurement of NIHR biomedical research centres in England. Res Eval. 2009;18(1):71-82.

65. Cole JR, Cole S. Social stratification in science. Chicago: The University of Chicago Press; 1973.

66. Shah SGS, Dam R, Milano MJ, Edmunds LD, Henderson LR, Hartley CR, et al. Gender parity in scientific authorship in a National Institute for Health Research Biomedical Research Centre: a bibliometric analysis. BMJ Open. 2021;11(3):e037935.

67. Crossref. You are crossref. 2020. https://www.crossref.org/. Accessed 24 Dec 2020.

68. European Science Foundation. Why plan S. 2018. https://www.coalition-s. org/why-plan-s/. Accessed 24 Dec 2020.

69. Van Raan A. The influence of international collaboration on the impact of research results. Scientometrics. 2006;42(3):423-8.

70. Dimensions Resources. Dimensions metrics API documentation. Dimensions. 2018. J Contrib. https://doi.org/10.6084/m9.figshare.5783694.v4.

71. The hidden REF. Submissions to REF2014. 2020. https://hidden-ref.org/ submissions-to-ref2014/. Accessed 24 Dec 2020.

72. Sprague ER. ORCID. J Med Libr Assoc. 2017;105(2):207-8.

73. Digital Science \& Research Solutions Ltd. GRID - global research identifier database. 2020. https://grid.ac/. Accessed 24 Dec 2020.

74. ROR (Research Organization Registry). Research Organization Registry Community. 2020. https://ror.org/. Accessed 24 Dec 2020. 
75. Fenner M. Powering the PID Graph: announcing the DataCite GraphQL API. DataCite Blog. 2020. https://blog.datacite.org/powering-the-pid-graph/. Accessed 24 Dec 2020.

76. Hook DW, Porter SJ, Herzog C. Dimensions: building context for search and evaluation. Front Res Metr Anal. 2018. https://doi.org/10.3389/frma.2018. 00023.

\section{List of Software: library/package (version) used.}

77. Python (3.7.6), Pandas (0.25.3), Networkx (2.4), Holoviews (1.12.7), Hvplot (0.5.2), Fuzzywuzzy (0.17.0), Habanero (0.7.2), Panel (0.7.0), Bokeh (1.4.0), ipython (7.11.1), Jupyter (1.0.0), notebook (6.0.2), numpy (1.17.5), scipy (1.3.2), selenium (3.141.0)

\section{Publisher's Note}

Springer Nature remains neutral with regard to jurisdictional claims in published maps and institutional affiliations.

- fast, convenient online submission

- thorough peer review by experienced researchers in your field

- rapid publication on acceptance

- support for research data, including large and complex data types

- gold Open Access which fosters wider collaboration and increased citations

- maximum visibility for your research: over 100M website views per year

At $\mathrm{BMC}$, research is always in progress.

Learn more biomedcentral.com/submissions 